\title{
The Relationship between Exercise and Athletes to the Pathogenesis and Recovery from Anorexia Nervosa
}

\author{
Ankita Vayalapalli1, ${ }^{1,}$, Rangaprakash Deshpande², Amoolya Vayalapalli ${ }^{1}$ \\ ${ }^{1}$ Northview High School, Georgia, USA \\ ${ }^{2}$ Department of Psychiatry and Biobehavioral Sciences, University of California, California, USA
}

Copyright $@ 2018$ by authors, all rights reserved. Authors agree that this article remains permanently open access under the terms of the Creative Commons Attribution License 4.0 International License

\begin{abstract}
Through two differing yet conjunctive perspectives, physical activity may be a factor amongst others that causes Anorexia Nervosa (AN), or a coping mechanism employed by AN patients to deal with the immense emotional and physical stress they experience. The brain's responses to exercise illustrate that both the putamen and the prefrontal cortex play an imperative role in the way human beings process and behaviorally respond to physical activity. In another case, the brain may turn to emotion suppression as a strategy to stave off or deal with the cognitive dissonance it experiences when using exercise as a coping outlet. This paper analyzes both perspectives through a neuroscience standpoint and is based largely on case studies and testimonials regarding the nature, progression, and recovery from AN in athletes and others in the physically active population.
\end{abstract}

Keywords Anorexia Nervosa, Exercise, Cognitive Reappraisal, Emotion Suppression, Sports Medicine, Neurobiological

\section{Introduction}

Anorexia nervosa (AN), a severe mental disorder that primarily afflicts young women, manifests through a distorted perception of one's body. It is characterized by an unusually low BMI (less than 18.5), rapid weight loss, food restriction, and compulsive exercise. It is estimated that approximately $2 \%$ of the United States population suffers from this disorder [2].Although this is true of the general population, AN presents itself in alarmingly higher rates within the sub-population of athletes and other physically active persons. The prevalence ranges from $12 \%$ to $70 \%$ in athletes, dependent on the sport [6]. The disparity is evident when comparing the rates of AN in endurance, aesthetic, and weight class sports to ball sports. Runners, gymnasts, and wrestlers are at an increased risk because of the nature of their sports-- the leaner body shape is favored and offers a competitive advantage over others. Analyzing the neurobiology behind the pathogenesis of AN in athletes is integral to decreasing and eventually eliminating the frequency of AN in athletes.

This paper analyzes the relationship between AN and the physically active population-- specifically athletes-- in two different lights. First, we present exercise as a coping mechanism that patients with AN employ to deal with the emotional stress of AN. Second, we present exercise as a factor in the pathogenesis of AN. In both scenarios, patients are likely to be compulsive exercisers and are at a high risk for other comorbid disorders such as anxiety, depression, and obsessive-compulsive disorder.

\section{Neurobiological Basis}

The prefrontal cortex enables decision making and processes complex thoughts and behaviors. The putamen, located at the base of the telencephalon (forebrain), controls the positive feedback loop of the limbs and is the body's primary response to intended physical activity [7]. In a study conducted at the University of Tubingen, Germany, the neurobiological responses to 'physical activity' and 'non-physical activity' and 'food' and 'nonfood' stimuli were recorded in a group of athletes with AN and a control group without AN [17]. The researchers concluded that both athletes with and without AN responded to the images with their somatosensory cortex, but upon further research the emotional aspect of the reactions were astoundingly different. When shown images of food, athletes with AN had "exaggerated response[s]" in the prefrontal cortex, the decision making hub of the brain. This concurs with anorexics' complex relationship with food-- they process it as a matter of great importance that requires complex thought and a complicated process of decision making. Furthermore, when shown images of physical activity, the putamen had a reduced inhibition response in athletes with AN rather than those without it. For anorexics, exercise or any form of physical activity requires less thinking-- it is a reflex rather than a carefully planned action. It is as second nature and requires less 
processing so because they feel it is a necessity and develops as a habit.

Exercise is associated with the release of dopamine and serotonin, the "feel good" hormones. They are known to reduce anxiety and promote overall well-being. Exercise can be defined as purposeful physical activity-- something which athletes engage in on a regular basis [8]. The relationship between athletes with $\mathrm{AN}$ and exercise can be analyzed before and after the pathogenesis of the disorder. Exercise can be viewed as either a risk factor that contributes to the development of AN or as a way to reduce the emotional stress caused by AN.

\section{Exercise as a Coping Mechanism}

Compulsive exercise can be defined as an "adherence to strict and recurring exercise routines maintained in spite of negative consequences" [10]. The Norwegian School of Sports Sciences investigated athletes with AN and their subjective relationships with exercise through testimonials and interview. One of the subjects in the study claimed that exercise, although "exhaustive and aversive", was something she felt drawn to because it seemed to be the only outlet for her stress. Her distorted body image frustrated her and caused her to stop eating and increase the intensity and frequency of exercise [33]. This counterintuitive measure demonstrates the illogical thinking that many athletes with AN experience.

The amygdala is the part of the brain that facilitates the basic necessities for survival, including sex and emotional reactions such as fear and anger. In a study conducted by the Flemish Institute, the amygdala was imaged before and after rats were subjected to 'non stressful' and 'stressful' situations [10]. The study revealed a myriad of issues that are onset by chronic stress: shortened length of dendrites in neurons in the central amygdaloid nucleus, decreased radial diffusion, reduced density of neurons, and dysmyelination of the axons (causing the message relayed to other cells to be transmitted less efficiently).

Neuroimaging reveals that pathological changes, such as AN, can lead to unpredictable changes in the transmission of a message from the axon and is also correlated with demyelination and disruption of the eigenvector (a vector quantity of a transmission that multiplies as it crosses synaptic clefts) that runs across a neural circuit [7]. Due to these neurobiological issues caused by stress induced from AN, compulsive exercise becomes an outlet for patients. It is also very convenient, because the main qualm $\mathrm{AN}$ patients have is with the size and shape of their bodies, and exercise is known to alter both.

Understanding the relationship between exercise and relapse rates in patients with $\mathrm{AN}$ is crucial in order to facilitate progress in the development of a treatment plan. A cohort study at the Parnassia Psychiatric Institute in The Netherlands examined relapse rates in a group of $\mathrm{AN}$ patients in rehab. After 18 months at a rehab facility, 30\% of the participants experienced a relapse, or a BMI of less than 18.5 following treatment [3]. This number is consistent amongst other studies and is alarmingly high for a relapse rate. One woman claimed that although she wanted to adhere to the treatment plan, immediately following her release from the rehabilitation center, she became "over active" and found it "extremely difficult to resist the need to do...some sort of physical activity" [18]. The mind-body disconnect is a common sentiment amongst the other participants and patients with AN. The cognitive-dissonance that occurs is a result of the emotion suppression that ensues [17]. Although patients know that exercising excessively following a diagnosis of AN is detrimental to their health, they continue their exercise regimen. The decision making process behind the rationalization points to unhealthy emotion regulation. The two main types of emotion regulation processes are cognitive reappraisal and emotion suppression [6]. Cognitive reappraisal is a strategy to reinterpret an "emotion-eliciting" situation and alters the situation to elicit less of an emotional impact. The latter, emotion suppression, is an especially problematic way to cope; the suppression inhibits the response or replaces the response with an emotionally less stressful response. In summary, cognitive reappraisal changes the way a stressful situation is viewed, whereas emotional suppression alters the response and behaviors as a reaction to the situation.

Neuroimaging evidence suggests that emotion suppression begins in the prefrontal cortex and has considerable involvement of the amygdala, and controls the activity of the limbic system [24]. This is similar to the findings from an earlier study testing the neural responses of patients diagnosed with AN. Due to these similarities, it can be concluded that these two parts of the brain work closely together and affect the behavioral and physical responses of a person with $\mathrm{AN}$ and cause dissonance in regards to exercise and recovering from AN. The multidimensional relationship between exercise and AN has its foundations on the signals received and interpreted by the neurons in the brain and the responses that are physically carried out [6]. Any disparity between the two represents the cognitive dissonance that is associated with many of the illogical behaviors that AN patients suffer from, such as food restriction, distorted body image, and excessive exercise.

\section{Exercise as Factor in the Pathogenesis of AN}

Through a different perspective, exercise can be viewed as a factor that leads towards the pathogenesis of AN rather than a coping mechanism. In a study conducted in Norway out of 522 athletes and 448 non-athletes, $18 \%$ of the athletes exhibited symptoms of AN, whereas only 5\% of the non-athletes presented symptoms associated with AN [28]. Sports can be divided between 'ball sports' 
(basketball, baseball, football), 'endurance sports' (long-distance or mid-distance running), 'aesthetic sports' (gymnastics), and 'weight class sports' (powerlifting, wrestling). These subtypes will be referenced but also studied as a whole to provide a wider perspective on the effect of exercise and training in each type of sport and sports in general.

Many athletes with AN exhibit neuropsychiatric symptoms including memory loss, poor concentration, and insomnia [18]. In addition, AN has comorbidity with generalized anxiety disorder (GAD) and depression; compulsive exercise affects a similar demographic (late teens to mid-twenties) and is common amongst those that have previously been or are diagnosed in conjunction with GAD, obsessive compulsive disorder (OCD), and depression [5]. The relationship between compulsive exercise and AN can be assessed as a cause and effect relationship because athletes regularly exercise and are required to do so in order to maintain or achieve a certain level of fitness. In some instances, unfortunately, their regimen can be more harmful than beneficial.

In many weight class sports, athletes will 'bulk' for extended periods of time, in hopes of gaining muscle and increase their body weight. In the months, weeks, or even days leading towards a competition or weigh-in, the athlete will then proceed to 'cut', or reduce their weight in compliance to their weight class. Because of this 'bulk' and 'cut' concept, it is very easy for weight-class athletes to exhibit erratic and anorexic behavior because of a reduced caloric intake and increased caloric output (due to exercise). Studies show that at least $70 \%$ of weight-class athletes will experience symptoms of AN at least once during their competitive season [27].

Similar situations occur in aesthetic sports as well. The pressure to succeed and 'look the look' is immense and can cause stressful situations that facilitate illogical behaviors. Long distance athletes are also at an increased risk for developing AN as a result of their sport, because being lean and 'skinny' offers them a competitive advantage. Although being leaner does make athletes run faster, when their BMI drops below 18.5 and their body starts shutting down from caloric restriction and compulsive exercising, lean bodies only prove to be detrimental to their athletic performance.

In one study titled, The Tale of Two Runners", two college runners, one mid-distance (male) and one long-distance (female), were studied over a period of 8 years to understand the pathology and nature of their AN [26]. In the case study, the athletes explained that their need to constantly exercise stemmed from a desire for control. Because of the culture of [their] sports, they felt that their body weight was hindering their performance even when it was not. They began exercising for several hours a day outside of their normal team training schedule and restricting their caloric intake. Coaches and specialists soon realized that they needed critical medical care and heir diagnosis and recovery from AN revolved around altering their exercise regimen. Although diet also played an integral role, exercise was the defining factor of their condition.

\section{Discussion}

The relationship between exercise and AN is a multidimensional one that differs based on each case, but its undeniable association calls for more research to aid in treatment and recovery from AN. The complex and secretive nature of living with AN continues to be a factor that makes it harder to obtain accurate data about the disorder. From the studies and research compiled, it can be reasonably concluded that exercise plays a defining role in the pathogenesis and recovery from AN, which places athletes at a particular vulnerability because of their lifestyle. Many other factors such as psychosocial disorders, genetic factors, and sex are also correlated with the prevalence of AN, but exercise and lifestyle choices are controllable (more so than the others) factors, which is why they should be studied further.

The pathogenesis of AN is a process that differs slightly in most patients. In some of the cases studied, exercise led to emotional stress, which resulted in unhealthy coping strategies such as developing AN. One of these strategies is known as emotion suppression, causes illogical responses and behaviors to situations that would otherwise elicit a different or supposedly 'normal' response. Unlike cognitive appraisal, a strategy that perceives a stressful situation in a new light, emotion suppression can cause a person to exercise even when they know that it will only harm them, feeding into their cognitive dissonance. This dissonance is the disparity between the logical and illogical behaviors exhibited that differ from what the brain intends to respond with.

The foil to the aforementioned scenario would be developing AN as a result of non-exercise related factors and then resorting to physical activity to combat the emotional tension. In both situations, exercise exacerbates the detriments to a person's health. Although exercise is widely known as a beneficial factor in terms of health both physically and emotionally, patients diagnosed with AN should take special precautions to inhibit a possible addiction or obsessiveness to exercise.

In order to combat AN in patients who also display symptoms of obsessive exercise, they should first be made aware of their lapse in judgement. With awareness comes progress because without understanding the long-term repercussions of their actions, it can be difficult to produce desirable results during recovery. In addition to awareness, patients need to develop a comfortable relationship with exercise and training. Instead of associating it with weight loss and a 'desirable' body shape, they should understand the spectrum of health and fitness. Exercise should be a 
means towards healthy living-- both physical, mental, and emotional. When one of these suffers, they should be able to recognize their struggle and be aware of the prophylactic measures and treatment options that are available. Furthermore, it is not the athlete's sole responsibility to seek help-- but rather a concern that should be shared within the sports medicine team. The coaches, parents, and administrators should recognize the signs and symptoms of AN and promote treatment and recovery options to rehabilitate athletes.

\section{Acknowledgements}

Special thanks to Ms. Ashley Ulrich of Northview High School for her editing which greatly improved the quality of the manuscript.

\section{REFERENCES}

[1] Anderluh, M. (2008). Faculty of 1000 evaluation for Timing and prediction of relapse in a transdiagnostic eating disorder sample. F1000 - Post-publication peer review of the biomedical literature. doi:10.3410/f.1108662.564750.

[2] Bauman, A. (2004). Health benefits of physical activity for older adults - epidemiological approaches to the evidence. Optimizing Exercise and Physical Activity in Older People, 1-25. doi:10.1016/b978-0-7506-5479-1.50004-2.

[3] Berends, T., Meijel, B. V., Nugteren, W., Deen, M., Danner, U. N., Hoek, H. W., \& Elburg, A. A. (2016). Rate, timing and predictors of relapse in patients with anorexia nervosa following a relapse prevention program: a cohort study. BMC Psychiatry, 16(1). doi:10.1186/s12888-016-1019-y.

[4] Carter, J. C., Mercer-Lynn, K. B., Norwood, S. J., Bewell-Weiss, C. V., Crosby, R. D., Woodside, D. B., \& Olmsted,

[5] Cook, B. J., \& Hausenblas, H. A. (2008). The Role of Exercise Dependence for the Relationship between Exercise Behavior and Eating Pathology. Journal of Health Psychology, 13 (4), 495-502. doi:10.1177/1359105308088520

[6] Craft, L. L., \& Perna, F. M. (2004). The Benefits of Exercise for the Clinically Depressed. The Primary Care Companion to the Journal of Clinical Psychiatry, 06(03), 104-111. doi:10.4088/pcc.v06n0301.

[7] Csordas, M. C., \& Panceri, C. (2017). Supervised physical exercise in adolescents with anorexia nervosa: systematic review. Revista Debates em Psiquiatria, Ano 7( $\left.\mathrm{N}^{\circ} 1\right), 16-22$. doi:10.25118/2236-918x-7-1-2

[8] Cutuli, D. (2014). Cognitive reappraisal and expressive suppression strategies role in the emotion regulation: an overview on their modulatory effects and neural correlates. Frontiers in Systems Neuroscience, 8. doi:10.3389/fnsys.2014.00175.
[9] Deter, H. (1992). The Anorexia Nervosa Symptom Score: A Multidimensional Tool for Evaluating the Course of Anorexia Nervosa. The Course of Eating Disorders, 40-52. doi:10.1007/978-3-642-76634-3_4.

[10] Drabant, E. M., Mcrae, K., Manuck, S. B., Hariri, A. R., \& Gross, J. J. (2009). Individual Differences in Typical Reappraisal Use Predict Amygdala and Prefrontal Responses. Biological Psychiatry, 65(5), 367-373. doi:10.1016/j.biopsych.2008.09.007.

[11] Etkin, A., \& Wager, T. D. (2007). Functional Neuroimaging of Anxiety: A Meta-Analysis of Emotional Processing in PTSD, Social Anxiety Disorder, and Specific Phobia. American Journal of Psychiatry, 164(10), 1476-1488. doi:10.1176/appi.ajp.2007.07030504.

[12] Freimuth, M., Moniz, S., \& Kim, S. R. (2011). Clarifying Exercise Addiction: Differential Diagnosis, Co-occurring Disorders, and Phases of Addiction. International Journal of Environmental Research and Public Health, 8(12), 4069-4081. doi:10.3390/ijerph8104069.

[13] Gruber, J., Kogan, A., Quoidbach, J., \& Mauss, I. B. (2013). Happiness is best kept stable: Positive emotion variability is associated with poorer psychological health. Emotion, 13(1), 1-6. doi:10.1037/a0030262.

[14] Hausenblas, H. A., \& Downs, D. S. (2002). How Much is Too Much? The Development and Validation of the Exercise Dependence Scale. Psychology \& Health, 17(4), 387-404. doi:10.1080/0887044022000004894.

[15] Hausenblas, H. A., \& Downs, D. S. (2002). Exercise dependence: a systematic review. Psychology of Sport and Exercise, 3(2), 89-123.

doi:10.1016/s1469-0292(00)00015-7.

[16] Joghtaie, M. T. (2013). Morphometric and volumetric study of caudate and putamen nuclei in normal individuals by MRI: Effect of normal aging, gender and hemispheric differences. Polish Journal of Radiology, 78(3), 7-14. doi:10.12659/pjr.889364.

[17] Keating, C., Tilbrook, A. J., Rossell, S. L., Enticott, P. G., \& Fitzgerald, P. B. (2012). Reward processing in anorexia nervosa. Neuropsychologia, 50(5), 567-575. doi:10.1016/j.neuropsychologia.2012.01.036.

[18] Kohn, N., Eickhoff, S., Scheller, M., Laird, A., Fox, P., \& Habel, U. (2015). Corrigendum to "Neural network of cognitive emotion regulation - An ALE meta-analysis and MACM analysis”. NeuroImage, 111, 631. doi:10.1016/j.neuroimage.2015.03.003.

[19] Kolnes, L. (2016). 'Feelings stronger than reason': conflicting experiences of exercise in women with anorexia nervosa. Journal of Eating Disorders, 4(1). doi:10.1186/s40337-016-0100-8.

[20] Kotbagi, G., Morvan, Y., Romo, L., \& Kern, L. (2017). Which dimensions of impulsivity are related to problematic practice of physical exercise? Journal of Behavioral Addictions, 6(2), 221-228. doi:10.1556/2006.6.2017.024.

[21] Leggio, M. G., Mandolesi, L., Federico, F., Spirito, F., Ricci, B., Gelfo, F., \& Petrosini, L. (2005). Environmental enrichment promotes improved spatial abilities and 
enhanced dendritic growth in the rat. Behavioural Brain Research, 163(1), 78-90. doi:10.1016/j.bbr.2005.04.009

[22] M. P. (2012). A prospective study of predictors of relapse in anorexia nervosa: Implications for relapse prevention. Psychiatry Research, 200(2-3), 518-523. doi:10.1016/j.psychres.2012.04.037

[23] Mowry, C., Pimentel, A., Sparks, E., \& Hanlon, B. (2013). Materials characterization activities for \%E2\%80\%9CTake Our Sons \& Daughters to Work Day\%E2\%80\%9D 2013. doi:10.2172/1096449

[24] Ohira, H., Nomura, M., Ichikawa, N., Isowa, T., Iidaka, T., Sato, A., ... Yamada, J. (2006). Association of neural and physiological responses during voluntary emotion suppression. NeuroImage, 29 (3), 721-733. doi:10.1016/j.neuroimage.2005.08.047

[25] Petzinger, G., Holschneider, D., Fisher, B., Mcewen, S., Kintz, N., Halliday, M., ... Jakowec, M. (2015). The Effects of Exercise on Dopamine Neurotransmission in Parkinson's Disease: Targeting Neuroplasticity to Modulate Basal Ganglia Circuitry. Brain Plasticity, 1(1), 29-39. doi:10.3233/bpl-150021

[26] Quatromoni, P. A. (2017). A Tale of Two Runners: A Case Report of Athletes' Experiences with Eating Disorders in College. Journal of the Academy of Nutrition and Dietetics, 117(1), 21-31. doi:10.1016/j.jand.2016.09.032

[27] Selby, E. A., Cornelius, T., Fehling, K. B., Kranzler, A., Panza, E. A., Lavender, J. M., ... Grange, D. L. (2015). A perfect storm: examining the synergistic effects of negative and positive emotional instability on promoting weight loss activities in anorexia nervosa. Frontiers in Psychology, 6. doi:10.3389/fpsyg.2015.01260

[28] Serpell, L., Teasdale, J. D., Troop, N. A., \& Treasure, J. (2004). The development of the P-CAN, a measure to operationalize the pros and cons of anorexia nervosa. International Journal of Eating Disorders, 36(4), 416-433. doi:10.1002/eat.20040

[29] Toulany, A., \& Katzman, D. K. (2014). Eating Disorders. The Female Athlete Triad, 111-129. doi:10.1007/978-1-4899-7525-6_7

[30] Vandereycken, W. (2006). Denial of illness in anorexia nervosa-a conceptual review: part 1 diagnostic significance and assessment. European Eating Disorders Review, 14(5), 341-351. doi:10.1002/erv.721

[31] Waller, G. (2016). Faculty of 1000 evaluation for Relapse from remission at two- to four-year follow-up in two treatments for adolescent anorexia nervosa. F1000 Post-publication peer review of the biomedical literature. doi:10.3410/f.725294972.793516508

[32] Watson, D., Clark, L. A., \& Tellegen, A. (1988) Development and validation of brief measures of positive and negative affect: The PANAS scales. Journal of Personality and Social Psychology, 54(6), 1063-1070. doi:10.1037//0022-3514.54.6.1063

[33] Zmijewski, C. F., \& Howard, M. O. (2003). Exercise dependence and attitudes toward eating among young adults. Eating Behaviors, 4(2), 181-195. doi:10.1016/s1471-0153(03)00022-9 